\title{
Charge Transport in Non-Irradiated and Irradiated Silicon Detectors
}

\author{
C. Leroy, P. Roy \\ Université de Montréal, Montréal (Québec) H3C 3J7, Canada \\ G. Casse, M. Glaser, E. Grigoriev, F. Lemeilleur \\ CERN, Geneva, Switzerland
}

\begin{abstract}
A model describing the transport of the carriers of the charge deposited in $n$ type silicon detectors by ionizing particles is presented. In order to reproduce the experimental current pulse responses induced by alpha and beta particles in nonirradiated and irradiated detectors up to fluences $(\Phi)$ much beyond the $n$ to $p$-type inversion, a n-type region 15 microns deep is introduced on the $\mathrm{p}^{+}$side of the diode. This model also gives mobilities decreasing linearily up to fluences of around $5 \cdot 10^{13}$ particles $/ \mathrm{cm}^{2}$, and beyond, converging to saturation values of about $1000 \mathrm{~cm}^{2} / \mathrm{Vs}$ and $450 \mathrm{~cm}^{2} / \mathrm{Vs}$ for electrons and holes, respectively. The charge carrier lifetime degradation, due to trapping with increased fluence, is responsible for a charge collection deficit for $\beta$ particles and for $\alpha$ particles found in agreement with direct CCE measurements.
\end{abstract}

\section{Introduction}

The electrical characteristics of n-type silicon detectors as a function of the particle fluence $(\Phi)$ can be extracted by modelling the transport of the carriers of the charge deposited by $\alpha$ or $\beta$ particles in non-irradiated and irradiated silicon detectors [1]. The model is used to fit the experimental signal-current pulse response (measured as a function of the collection time) induced by $\alpha$ and $\beta$ particles in $p^{+}-n-n^{+}$diodes. The extracted electrical characteristics of a $p^{+}-n-n^{+}$diode are the effective impurity or dopant concentrations $\left(N_{e f f}\right)$, the electron $\left(\mu_{e}\right)$ and hole $\left(\mu_{h}\right)$ mobilities, and the charge carrier lifetimes $\left(\tau_{t e}\right.$, $\left.\tau_{t h}\right)$. 


\section{Charge transport model}

The electrical characteristics are extracted by solving in one-dimension (through the use of a transverse diffusion term) a system of five partial differential equations (ref. [1] for more details): the current continuity equations for electrons and holes, the Poisson equation (which determines the electric field and considers the plasma effects) and two equations relating the concentration of trapped to untrapped charges. A planar semiconductor $\left(p^{+}-n-n^{+}\right.$diode) is considered (Fig. 1), with the ohmic side and the junction side located at $x=0$ and $x=w$, respectively. When neglecting the size of the $p^{+}$and $n^{+}$ regions, the integration of the one-dimensional Poisson's equation at $t=0$, for a simple abrupt pn junction operated in overdepleted mode $\left(V_{b}>V_{d}\right)$, gives:

$$
\begin{gathered}
E(x, 0)=-\frac{q N_{\mathrm{eff}}}{\epsilon} x+\frac{V_{b}}{w}+\frac{V_{d} N_{\mathrm{eff}}}{w\left|N_{\mathrm{eff}}\right|} \quad \text { for } 0<x<w \\
E(0, t)=E(w, t)=0 \\
\psi(0, t)=V_{0}+\psi_{p} \approx 0 \quad \text { and } \quad \psi(w, t)=\psi_{p}-V_{b} \approx-V_{b}
\end{gathered}
$$

where $\psi$ and $E$ are the electrostatic potential and the electric field, respectively; $\mathrm{q}, \epsilon$ and $w$ are the electrical charge, the permittivity and the thickness of the diode, respectively; $V_{0} \approx .6$ volt is the built-in voltage, $V_{b}$ the applied bias voltage, $\psi_{p} \approx-.3$ volt the electrostatic potential of the neutral p-type region and $V_{d}$ is the full depletion bias voltage.

The drift velocity of the charge carrier reaches a saturation value $v_{s}$ for electric field values around $10^{4} \mathrm{~V} / \mathrm{cm}$. The empirical equation describing the mobility as a function of the electric field is:

$$
\mu(x)=\frac{\mu_{0}}{\left[1+\left(\mu_{0} E(x) / v_{s}\right)^{m}\right]^{1 / m}}
$$

where $\mu_{0}$ is the zero field mobility, $m=1$ for holes, $m=2$ for electrons; $v_{s}=$ $1.05 \cdot 10^{7}$ and $10^{7} \mathrm{~cm} / \mathrm{s}$ for electrons and holes, respectively.

The mobilities are also dependent on the temperature and dopant concentrations. Changing the temperature by 1.5 degree Celsius changes both mobilities by $\approx 1 \%$. In the present work, all the measurements using $\alpha$ particles and several using $\beta$ particles were performed at room temperature $\left(22^{\circ} \mathrm{C}\right)$, while the measurements using $\beta$ particles for detectors P88, P189 and P304 were made at $15^{\circ} \mathrm{C}$. The temperature and the dopant concentration dependence of mo- 
bility is taken into account via the empirical equation:

$$
\mu\left(T, N_{e f f}\right)=\mu_{m i n}+\frac{\mu_{0}\left(\frac{T}{300}\right)^{\nu}-\mu_{\min }}{1+\left(\frac{T}{300}\right)^{\xi}\left(\frac{N_{e f f}}{N_{\text {ref }}}\right)^{\alpha}}
$$

where the values used for the electrons (holes) are: $\mu_{\min }=55.24(49.7) \mathrm{cm}^{2} / \mathrm{Vs}$, $N_{\text {ref }}=1.072 \cdot 10^{17}\left(1.606 \cdot 10^{17}\right)$ dopants $/ \mathrm{cm}^{3}, \nu=-2.3(-2.2), \xi=-3.8(-3.7)$, $\alpha=0.73(0.70), T$ is the temperature in Kelvin and $\mu_{0}$ is the mobility at $T$ $=300 \mathrm{~K}$. The temperature dependence of mobility is shown in Fig. 2 using $\mu_{0}($ for $\mathrm{e})=1350 \mathrm{~cm}^{2} / \mathrm{Vs}$ and $\mu_{0}($ for $\mathrm{h})=480 \mathrm{~cm}^{2} /$ Vs at $T=300 \mathrm{~K}$.

In absence of an analytical solution to the system of the five partial differential equations, the equations are discretized using Gummel's decoupling scheme [2] to obtain a numerical solution [1]. The observed signal $(V(t))$ is a convolution of the current $(I(t)$ obtained from Ramo's theorem [3]) produced by all the individual charge carriers and the response from the system, which is simply an $\mathrm{RC}$ circuit. The response of the system is a Gaussian with a characteristic time constant $\sigma=R_{a} C$, where $C$ is the capacitance of the detector and $R_{a}=50 \Omega$ the input impedance of the amplifier:

$$
\begin{aligned}
I(t) & =\frac{18 D_{a} t+r_{0}^{2}}{w r_{0}^{2}} \int_{0}^{w}\left(\mu_{e} n+\mu_{h} p\right) E d x \\
V(t) & =\frac{G R_{a}}{\sigma \sqrt{2 \pi}} \sum_{e, h} \int_{-\infty}^{\infty} I\left(t^{\prime}\right) \exp \left(-\frac{\left(t-t^{\prime}\right)^{2}}{2 \sigma^{2}}\right) d t^{\prime}
\end{aligned}
$$

where $D_{a}$ is the ambipolar diffusion constant, $G=1000$ the gain of the amplifier and $r_{0}$ the initial radius of the column of deposited charge.

The quantities of interest are extracted by using the code MINUIT [4] to minimize the $\chi^{2}$ obtained from fitting the numerical solutions found for $\mathrm{V}(\mathrm{t})$ to the measured current pulse response induced by $\alpha$ particles from a ${ }^{241} \mathrm{Am}$ source with an energy of $5.49 \mathrm{MeV}$ and $\beta$ particles from a ${ }^{106} \mathrm{Ru}$ source with an energy $>2 \mathrm{MeV}$, selected by an external trigger. The current pulses induced by particles penetrating the silicon diode are detected by a fast current amplifier. The pulses are recorded by a LeCroy digital oscilloscope used in averaging mode, to improve the signal-to-noise ratio $[5,6]$. A summary of the characteristics, at $\Phi=0$, of the standard float zone silicon detectors used in the present work is given in Table 1 . 


\subsection{Non-irradiated detectors $(\Phi=0)$}

Fits of the charge carriers transport model to the current pulses induced by relativistic electrons and by $\alpha$-particles incident either on the front-side or the back-side of non-irradiated detectors $(\Phi=0)$ reproduces well the shape of the measured current pulses $(\alpha$ and $\beta$ ) as shown in Fig. 3 (detectors M25 and M50 as examples). The model also gives the values of the electrons and holes mobilities reported in Table 2. The average mobilities achieved for electrons and holes at $\Phi=0$ for the detectors described in Table 1 are: $\mu_{h}=491 \pm 10$ $\mathrm{cm}^{2} / \mathrm{Vs}$ and $\mu_{e}=1267 \pm 20 \mathrm{~cm}^{2} / \mathrm{Vs}$, respectively.

\subsection{Irradiated detectors}

The detectors have been irradiated either with $\approx 1 \mathrm{MeV}$ neutrons (at the CERN PSAIF) up to a fluence of $9.92 \times 10^{13} \mathrm{n} / \mathrm{cm}^{2}$ or with $24 \mathrm{GeV} / \mathrm{c}$ protons (at the CERN PS) up to a fluence of $2.87 \times 10^{14} \mathrm{p} / \mathrm{cm}^{2}$ (column 6 in Table 1 ).

The results of the charge transport model fits to the experimental data permit the extraction of the value of $N_{\text {eff }}$ as a function of fluence according to:

$$
\mathbf{N}_{\text {eff }}=\mathbf{N}_{0} \exp (-c \Phi)+b \Phi
$$

By using Eq. (8) to describe the evolution of $N_{\text {eff }}$ with fluence, one obtains the results reported in Table 3 . The difference between $N_{\text {eff reported in Table } 1}$ and $N_{0}$ of Table 3 is due to the fact that the former value is obtained from the specifications given by the manufacturers, while the later is the fitted value (Eq. (8)). The results reported in Table 3 show that a silicon detector, initially of n-type, becomes apparently intrinsic around $\Phi_{i n v}=5 \times 10^{12}$ particles $\mathrm{cm}^{-2}$ ( $\Phi_{i n v}$ is the value of the fluence at inversion) and inverts to an apparent p-type for higher fluences, with $N_{\text {eff }}$ (Eq. (8)) increasing with fluence. It has to be observed that correlated with the conduction-type inversion, the junction moves from the front side to the back side. As a consequence, for a given bias voltage, the shape of the electric fields depends on the fluence level (Eq. (1)) as does the current induced by the moving charge carriers. The current slope is negative before irradiation, decreases up to $\Phi_{i n v}$, changes sign with the conduction-type inversion, and becomes more and more positive with increasing fluence [1]. 


\subsection{1 $\alpha$ particles data}

Using a simple $\mathrm{pn}^{+}$junction after the $\mathrm{n}$ to $\mathrm{p}$-type inversion (which takes place at a fluence $\Phi \equiv \Phi_{i n v} \approx 5 \times 10^{12}$ particles $/ \mathrm{cm}^{2}$, a detector with an higher initial resistivity inverts from $n$ to $p$-type at lower fluence value), the charge carriers transport model poorly reproduces the measured $\mathrm{V}(\mathrm{t})$ as a function of collection time for $\Phi>\Phi_{i n v}$ as shown in Fig. 4a-c for $\alpha$ particles incident on the back side of detector M4.

In order to fit the data and to account for the evolution of the electrical characteristic of the detectors for fluence beyond $\Phi_{i n v}$, the electric field is modified by introducing a $15 \mu \mathrm{m}$ n-type region near the $p^{+}$contact. This concept of double junction can be also found in [7]. This modification of the electric field permits now to reproduce the measured $V(t)$ as a function of collection time for $\Phi>\Phi_{i n v}$, as shown in Fig. 4d-f for $\alpha$ particles incident on the back side of detector M4. Another example of results of the fits of the charge transport model for $\alpha$ particles incident on the front and back side of detector M25 are shown in Fig. 5 and 6.

The electrons and holes mobilities extracted from the $\alpha$ particles data, after an initial linear decrease with increasing fluence, tends towards a saturation value $\left(\mu_{s a t_{e, h}}\right)$ for fluences beyond $\approx 5 \cdot 10^{13}$ particles $/ \mathrm{cm}^{2}$. The initial decrease is described by:

$$
\mu_{e, h}=a_{e, h}-b_{e, h} \times \Phi
$$

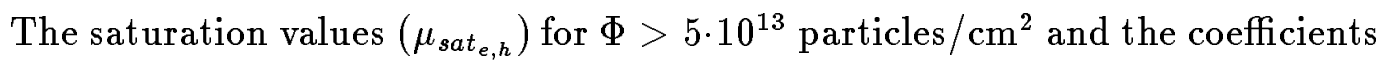
$a_{e, h}$ and $b_{e, h}$ are given in Table 4 for $\alpha$ particles data.

\subsection{2 $\beta$ particles data}

The charge transport model was also tested using data obtained from the current pulse response induced by $\beta$ particles on the non-irradiated (section 2.1) and irradiated detectors of Table 1. The results of the fits of the charge transport model are shown in Fig. 7 for detector M25 for successive levels of fluence $\Phi$ up to $7.5 \times 10^{13} \mathrm{p} / \mathrm{cm}^{2}$.

The mobility extracted from the $\beta$ data are reported in Table 4 . A comparison of the mobility values extracted from $\alpha$ and $\beta$ data is extended in Figs. 8ab. These figures show that the mobilities (for electrons and holes) extracted from $\alpha$ and $\beta$ data are found to be in very good agreement, which provides a consistency check of the model. 


\subsection{Charge collection efficiency}

As the fluence increases and thus the number of traps, the charge carrier lifetimes due to trapping are found to be decreasing. The integration of $\mathrm{V}(\mathrm{t})$ over the collection time allows one to determine the collected charge, and thus by comparison with the results obtained using the trapping lifetime extracted at the maximum fluences $\left(\Phi \approx 10^{14}\right.$ particles $\left./ \mathrm{cm}^{2}\right)$ with those obtained if no trapping had occured, it is possible to calculate the charge collection deficit. For neutron (proton) irradiated detectors, a charge collection deficit around $12 \%(20 \%)$ is calculated for $\alpha$ particles incident on the front side and about $18 \%(30 \%)$ for $\alpha$ particles incident on the back side of the detector, for a fluence of $\approx 10^{14}$ particles $/ \mathrm{cm}^{2}$. For $\beta$ particles, a collection deficit of about $13 \%$ is calculated for a fluence of $\approx 10^{14}$ particles $/ \mathrm{cm}^{2}$. This latter result is in agreement with the $12 \%$ deficit obtained from direct charge collection efficiency (CCE) measurements made with $\beta$ particles (shaping time of 100 ns) [8] for detectors irradiated at fluences $\approx 10^{14}$ particles $/ \mathrm{cm}^{2}$. Direct measurements of CCE made with $\alpha$ particles at a fluence of $\approx 10^{14}$ protons $/ \mathrm{cm}^{2}$ show a smaller deficit $(\approx 5 \%$ on the front side and $\approx 10 \%$ on the back side $[9])$, but these data were recorded with a shaping time $(1 \mu \mathrm{s})$ larger than the shaping time $(100 \mathrm{~ns})$ used in the present work.

\section{Conclusion}

A model describing the transport of the carriers of the charge deposited in ntype silicon detectors by ionizing particles has been presented. To reproduce the experimental current pulse responses induced by alpha and beta particles in non-irradiated and irradiated detectors up to fluences much beyond the $n$ to p-type inversion, a n-type region 15 microns deep was introduced on the $\mathrm{p}^{+}$side of the diode. The introduction of this region modifies the electric field after inversion and permits the charge carriers transport model to reproduce the experimental data up to available fluences of $3 \cdot 10^{14}$ particles $/ \mathrm{cm}^{2}$.

Using this model, the mobilities extracted from data collected with $\alpha$ particles incident on the front and back side of the detectors are found to be decreasing linearily with increasing fluence up to fluences of around $5 \times 10^{13}$ particles $/ \mathrm{cm}^{2}$, and beyond, converging to saturation values of about $1000 \mathrm{~cm}^{2} / \mathrm{Vs}$ and 450 $\mathrm{cm}^{2} / \mathrm{Vs}$ for electrons and holes, respectively. The mobility extracted from the $\beta$ data for the same detectors are found to be in very good agreement with the mobilities extracted from data collected with $\alpha$ particles. This agreement provides a consistency check of the model.

The charge carrier lifetime degradation due to trapping with increased fluence 
is responsible for a charge collection deficit and found to be in agreement with the charge collection deficit measured directly with $\beta$ and $\alpha$ particles.

\section{References}

[1] C. Leroy et al., Study of Charge Transport in Non-Irradiated and Irradiated Silicon Detectors, International Conference on Radiation Effects on Semiconductor Materials, Detectors and Devices, Florence, Italy, March 1998, to be published in Nucl. Inst. and Meth. A.

[2] H. K. Gummel, A self-consistent iterative scheme for one-dimensional steady state transistor calculations, IEEE Trans. Electron. Devices ED-11 (1964) 455.

[3] S. Ramo, Currents induced by electron motion, Proc. IRE vol. 27(9) (1939) 584.

[4] Application Software Group, CERN Program Library D506, MINUIT Function Minimization and Error Analysis, 1992.

[5] P. Roy, Étude de la réponse en courant de détecteur silicium opérés en environnement de très hautes radiations, M.Sc. thesis, Université de Montréal, 1994.

[6] C. Leroy et al., Study of charge collection and noise in non-irradiated and irradiated silicon detectors, Nucl. Inst. and Meth. A 388 (1997) 289.

[7] Z. Li and H.W. Kraner, Fast neutron radiation damage effects on high resistivity silicon junction detectors, Journ. of Elec. Mat. vol. 21(7) (1992) 701.

[8] C. Leroy et al., Study of electrical properties and charge collection of silicon detectors under neutron, proton and gamma irradiations, Proc. IVth Int. Conf. on Calorimetry in High Energy Physics, La Biodola, Isola d'Elba, Italy, 19-25 september 1993, World Scientific, eds. A. Menzione and A. Scribano, Singapore (1994) 627 .

[9] S. Pospisil, talk given at this conference. 


\section{Table caption}

Table 1: Characteristics at $\Phi=0$ of the standard float zone detectors used in the present work. The detectors have been irradiated by step of fluence from $\Phi=0$ up to $9.92 \times 10^{13} \mathrm{n} / \mathrm{cm}^{2}$ for M4, up to $7.5 \times 10^{13} \mathrm{p} / \mathrm{cm}^{2}$ for M18, M25, $\mathrm{M} 35$, and up to $28.7 \times 10^{13} \mathrm{p} / \mathrm{cm}^{2}$ for P88, P189 and P304. M49, M50, M53 were not irradiated.

Table 2: Electron and hole mobilities of the detectors, listed in Table 1, extracted from the model fitted to $\beta$ and $\alpha$ data at $\Phi=0$.

Table 3: Parameters describing the evolution of the effective dopant concentration $\left(N_{e f f}\right)$ with fluence $(\phi)$.

Table 4: Parameters describing the evolution of the mobilities with fluence as extracted from data obtained by $\alpha$ particles incident on the front side, on the back side and by $\beta$ particles (eq. (9) applied to $\alpha$ and $\beta$ data). 


\section{Figure caption}

Fig. 1: a) Representation of a $p^{+}-n-n^{+}$diode, b) the dopant profile $\left.(\rho), \mathrm{c}\right)$ the electric field $(E)$ and d) the electrostatic potential $(\psi)$.

Fig. 2: Effect of the temperature on the mobilities (according to eq. (5), with $\mu_{0}($ for $\mathrm{e})=1350 \mathrm{~cm}^{2} / \mathrm{Vs}$ and $\mu_{0}($ for $\mathrm{h})=480 \mathrm{~cm}^{2} / \mathrm{Vs}$ at $\left.T=300 \mathrm{~K}\right)$.

Fig. 3: Fits (full line) of the charge transport model to the current pulse response induced at $\Phi=0$ by $\alpha$ particles incident on the front side (a), on the back side (b) of detector M25 and by relativistic electrons on detector M50 (c); A bias voltage $V_{b}=160 \mathrm{~V}$ is applied in all cases. The individual electrons (e) and holes (h) contributions are shown.

Fig. 4: Fits (full line) of the current pulse response induced by $\alpha$ particles incident on the back side of detector M4: before (a, b and c) and after ( $d$, e and $\mathrm{f}$ ) the introduction of the $15 \mu \mathrm{m}$ n-type junction on the $p^{+}$side after inversion at fluences of $\Phi=6.27(\mathrm{a}, \mathrm{d}), 7.46(\mathrm{~b}, \mathrm{e})$ and $9.92 \times 10^{13} \mathrm{n} / \mathrm{cm}^{2}(\mathrm{c}, \mathrm{f})$. The bias voltage was 160 volts.

Fig. 5: Fits (full line) of the current pulse response induced by $\alpha$ particles incident on the front side of detector M25 for successive levels of fluence $\Phi$ from 0 up to $4.95 \times 10^{13} \mathrm{p} / \mathrm{cm}^{2}$ ( $\Phi$ is in units of $10^{13} \mathrm{p} / \mathrm{cm}^{2}, V_{b}$ is the applied voltage in volts).

Fig. 6: Fits (full line) of the current pulse response induced by $\alpha$ particles incident on the back side of detector M25 for successive levels of fluence $\Phi$ from 0 up to $7.5 \times 10^{13} \mathrm{p} / \mathrm{cm}^{2}$ ( $\Phi$ is in units of $10^{13} \mathrm{p} / \mathrm{cm}^{2}, V_{b}$ is the applied voltage in volts).

Fig. 7: Fits (full line) of the current pulse response induced by $\beta$ particles incident on detector M25 for successive levels of fluence $\Phi$ from 0 up to $7.5 \times$ $10^{13} \mathrm{p} / \mathrm{cm}^{2}$ ( $\Phi$ is in units of $10^{13} \mathrm{p} / \mathrm{cm}^{2}, V_{b}$ is the applied voltage in Volts).

Fig. 8: Comparison of the electrons (a) and holes (b) mobilities extracted from $\alpha$ and $\beta$ particles data using detectors M18, M25, P88, P189 and P304. 


\begin{tabular}{|l|c|c|c|c|c|}
\hline Detector & $\begin{array}{c}\text { Current } \\
\text { pulse source }\end{array}$ & $\begin{array}{c}\text { thickness } \\
(\mu \mathrm{m})\end{array}$ & $\begin{array}{c}\mathrm{N}_{\text {eff }} \\
\left(10^{11} \mathrm{~cm}^{-3}\right)\end{array}$ & $\begin{array}{c}\rho \\
\mathrm{k} \Omega \times \mathrm{cm}\end{array}$ & $\begin{array}{c}\text { Maximum } \\
\text { fluence }\left(\mathrm{cm}^{-2}\right)\end{array}$ \\
\hline M4 $\left(1 \mathrm{~cm}^{2}\right)$ & $\alpha$ & 317 & -3.4 & 12.2 & $9.92 \times 10^{13} \mathrm{n}$ \\
\hline M18 $\left(1 \mathrm{~cm}^{2}\right)$ & $\alpha, \beta$ & 309 & -4.1 & 11. & $7.5 \times 10^{13} \mathrm{p}$ \\
\hline M25 $\left(1 \mathrm{~cm}^{2}\right)$ & $\alpha, \beta$ & 308 & -2.1 & 23. & $7.5 \times 10^{13} \mathrm{p}$ \\
\hline M35 $\left(1 \mathrm{~cm}^{2}\right)$ & $\alpha$ & 508 & -1.7 & 24. & $7.5 \times 10^{13} \mathrm{p}$ \\
\hline M49 $\left(1 \mathrm{~cm}^{2}\right)$ & $\beta$ & 301 & -4.7 & 8.9 & - \\
\hline M50 $\left(1 \mathrm{~cm}^{2}\right)$ & $\beta$ & 471 & -1.8 & 22.8 & - \\
\hline M53 $\left(1 \mathrm{~cm}^{2}\right)$ & $\beta$ & 223 & -5.4 & 7.7 & - \\
\hline P88 $\left(.25 \mathrm{~cm}^{2}\right)$ & $\alpha, \beta$ & 290 & -18 & 2.5 & $28.7 \times 10^{13} \mathrm{p}$ \\
\hline P189 $\left(.25 \mathrm{~cm}^{2}\right)$ & $\alpha, \beta$ & 294 & -18 & 2.5 & $28.7 \times 10^{13} \mathrm{p}$ \\
\hline P304 $\left(.25 \mathrm{~cm}^{2}\right)$ & $\alpha, \beta$ & 320 & -7 & 6. & $28.7 \times 10^{13} \mathrm{p}$ \\
\hline
\end{tabular}

Table 1 


\begin{tabular}{|l|c|l|l|}
\hline Detector & $\begin{array}{c}\text { Current } \\
\text { pulse source }\end{array}$ & $\begin{array}{l}\mu_{h} \\
\left(\mathrm{~cm}^{2} \times \mathrm{V}^{-1} \times \mathrm{s}^{-1}\right)\end{array}$ & $\begin{array}{l}\mu_{e} \\
\left(\mathrm{~cm}^{2} \times \mathrm{V}^{-1} \times \mathrm{s}^{-1}\right)\end{array}$ \\
\hline M4 & $\alpha$ & $503.8 \pm 2.2$ & $1278 \pm 15$ \\
\hline M18 & $\alpha, \beta$ & $474.4 \pm 2.4$ & $1236 \pm 15$ \\
\hline M25 & $\alpha$ & $476.0 \pm 2$ & $1308 \pm 28$ \\
\hline M35 & $\alpha$ & $472.1 \pm 3$ & $1272 \pm 5$ \\
\hline M49 & $\beta$ & $546 \pm 11$ & $1266 \pm 24$ \\
\hline M50 & $\beta$ & $529 \pm 13$ & $1272 \pm 20$ \\
\hline M53 & $\beta$ & $478 \pm 12$ & $1350 \pm 20$ \\
\hline P88 & $\alpha$ & $459.1 \pm 4$ & $1222 \pm 20$ \\
\hline P189 & $\alpha$ & $480 \pm 20$ & $1340 \pm 27$ \\
\hline P304 & $\alpha$ & $495 \pm 3$ & $1124 \pm 22$ \\
\hline
\end{tabular}

Table 2 


\begin{tabular}{|l|c|c|c|c|}
\hline Detector & $\mathrm{N}_{0}$ & $b$ & $c$ & $\Phi_{i n v}$ \\
\hline & $10^{11} \mathrm{~cm}^{-3}$ & $10^{-2} /($ particles cm) & $10^{-11} \mathrm{~cm}^{2} /$ particles & $10^{12} \mathrm{~cm}^{-2}$ \\
\hline M4 & -2.7 & 1.4 & 9 & $\approx 8 . \mathrm{n}$ \\
\hline M18 & -3.7 & 2.8 & 10 & $\approx 7 . \mathrm{p}$ \\
\hline M25 & -2.2 & 2.5 & 85 & $\approx 2 . \mathrm{p}$ \\
\hline M35 & -2.2 & 2.7 & 76 & $\approx 2 . \mathrm{p}$ \\
\hline
\end{tabular}

Table 3 


\begin{tabular}{|l|c|c|c|c|c|c|}
\hline Detector & $a_{h}$ & $a_{e}$ & $b_{h}$ & $b_{e}$ & $\mu_{\text {sat }_{h}}$ & $\mu_{\text {sat }}$ \\
\hline & $\mathrm{cm}^{2} / \mathrm{Vs}$ & $\mathrm{cm}^{2} / \mathrm{Vs}$ & $\mathrm{cm}^{4} /($ particles Vs $)$ & $\mathrm{cm}^{4} /($ particles Vs $)$ & $\mathrm{cm}^{2} / \mathrm{Vs}$ & $\mathrm{cm}^{2} / \mathrm{Vs}$ \\
\hline M4 $(\alpha)$ & 504 & 1200 & 5.6 & 46 & 455 & 990 \\
\hline M18 $(\alpha)$ & 474 & 1195 & 5.3 & 124 & 460 & 1000 \\
\hline M25 $(\alpha)$ & 473 & 1305 & 6.3 & 121 & 460 & 990 \\
\hline M35 $(\alpha)$ & 490 & 1233 & 8 & 115 & 460 & 1000 \\
\hline P88 $(\alpha)$ & 456 & 1176 & 1.6 & 18 & 440 & 1000 \\
\hline P189 $(\alpha)$ & 474 & 1280 & 3.0 & 34 & 450 & 980 \\
\hline P304 $(\alpha)$ & 499 & 1252 & 4.4 & 17 & 452 & 1100 \\
\hline M18 $(\beta)$ & 481 & 1187 & 14. & 107 & 460 & 1000 \\
\hline M25 $(\beta)$ & 496 & 1287 & 12 & 113 & 470 & 990 \\
\hline P88 $(\beta)$ & 428 & 1154 & 3.1 & 7.6 & 395 & 1070 \\
\hline P189 $(\beta)$ & 460 & 1286 & 0.5 & 23 & 450 & 1050 \\
\hline P304 $(\beta)$ & 431 & 1270 & 0.3 & 18 & 425 & 1100 \\
\hline
\end{tabular}

Table 4 

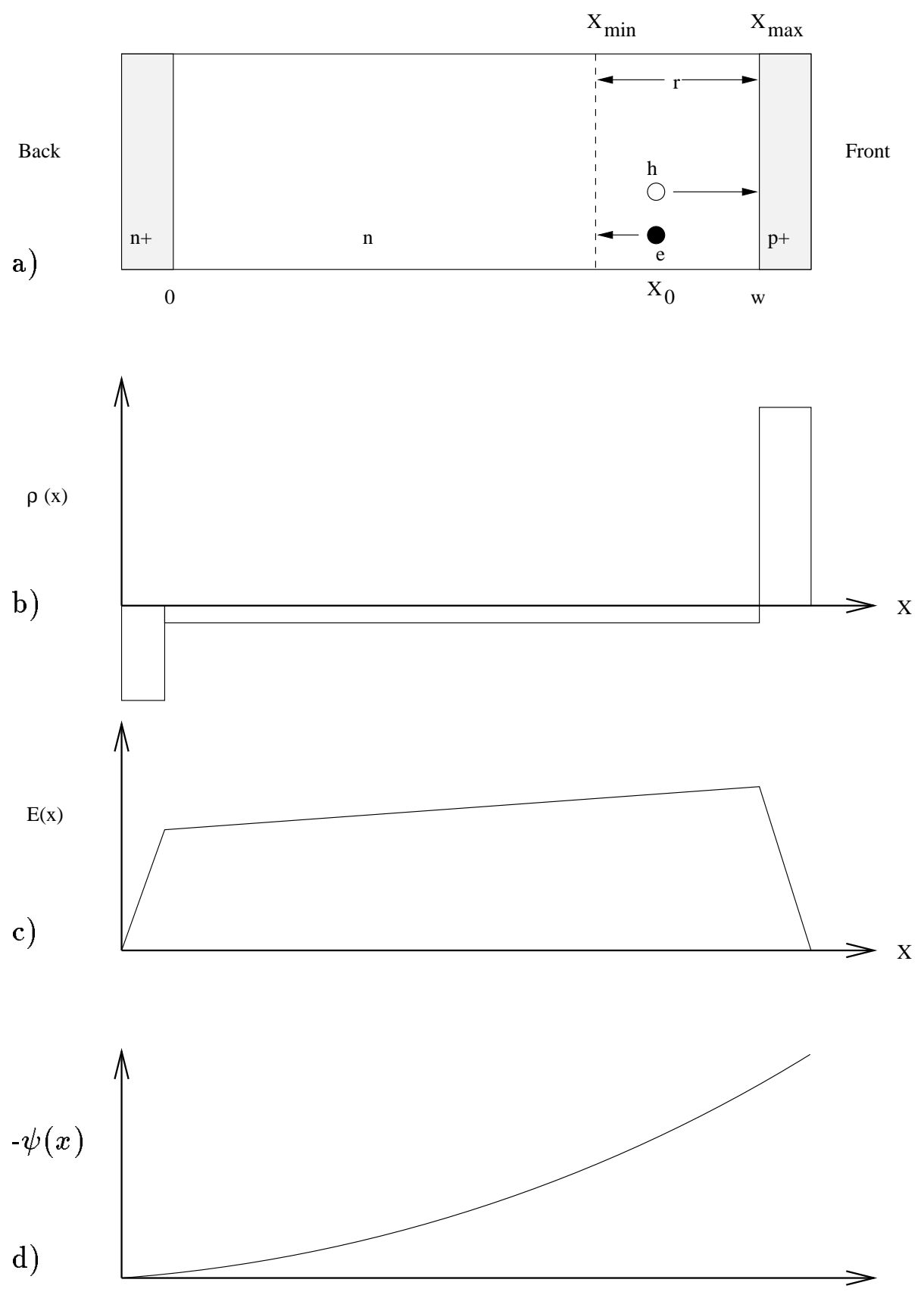

Fig. 1. 

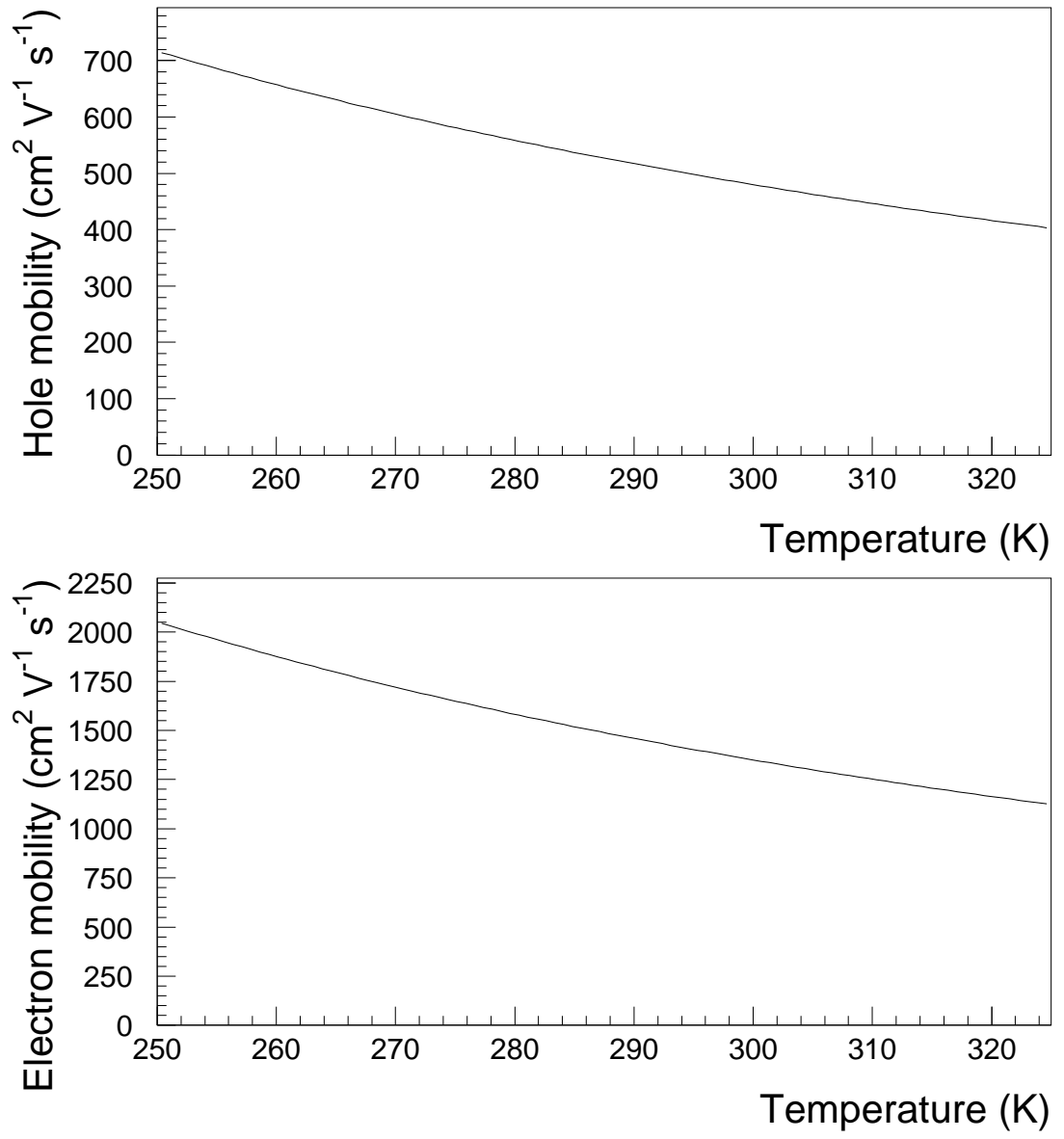

Fig. 2. 

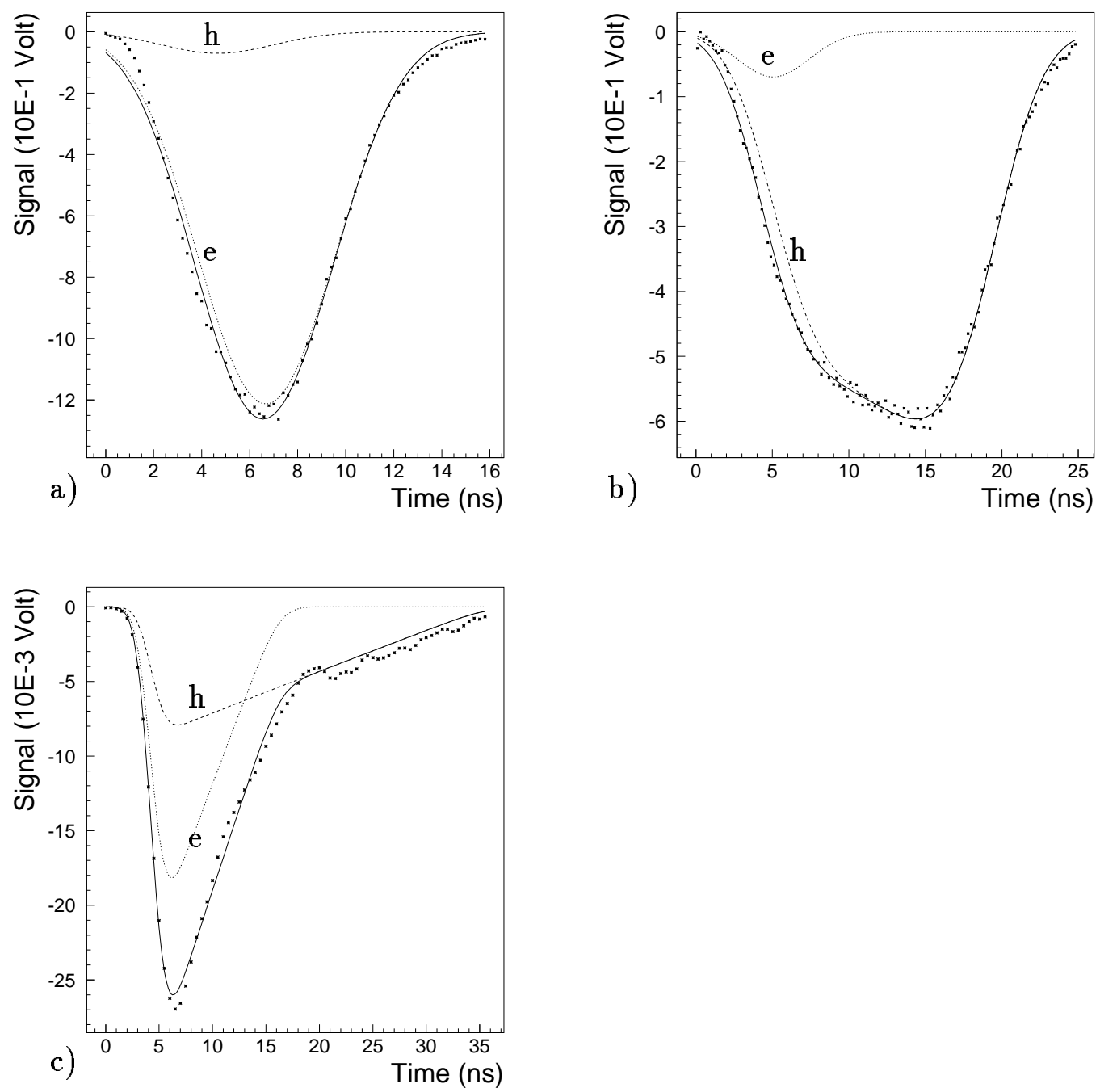

Fig. 3. 
Without double junction
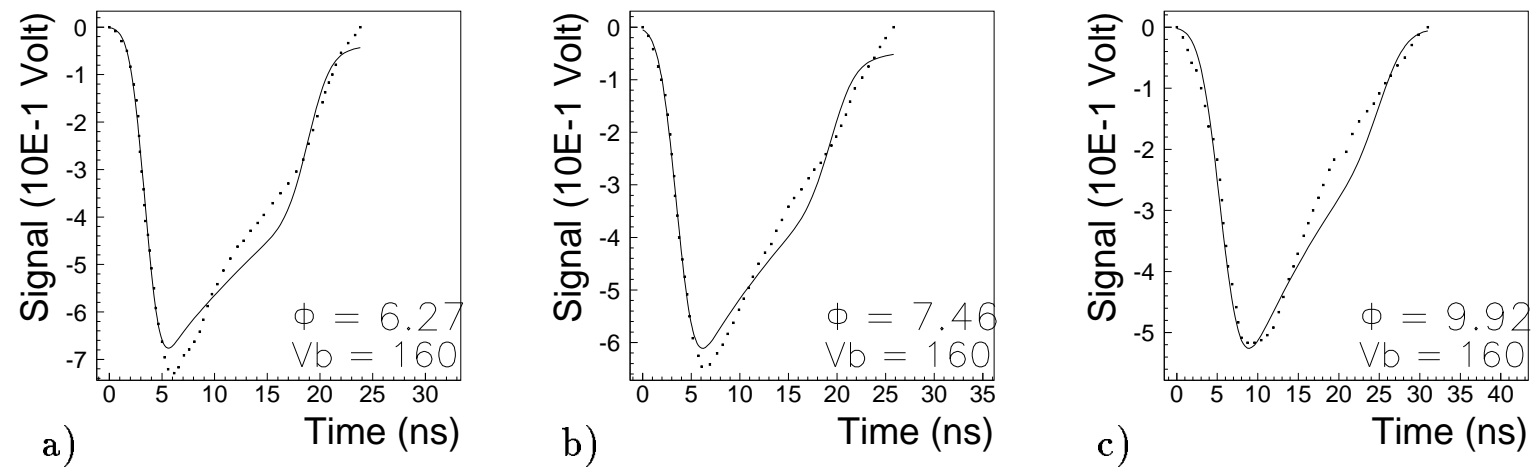

With double junction
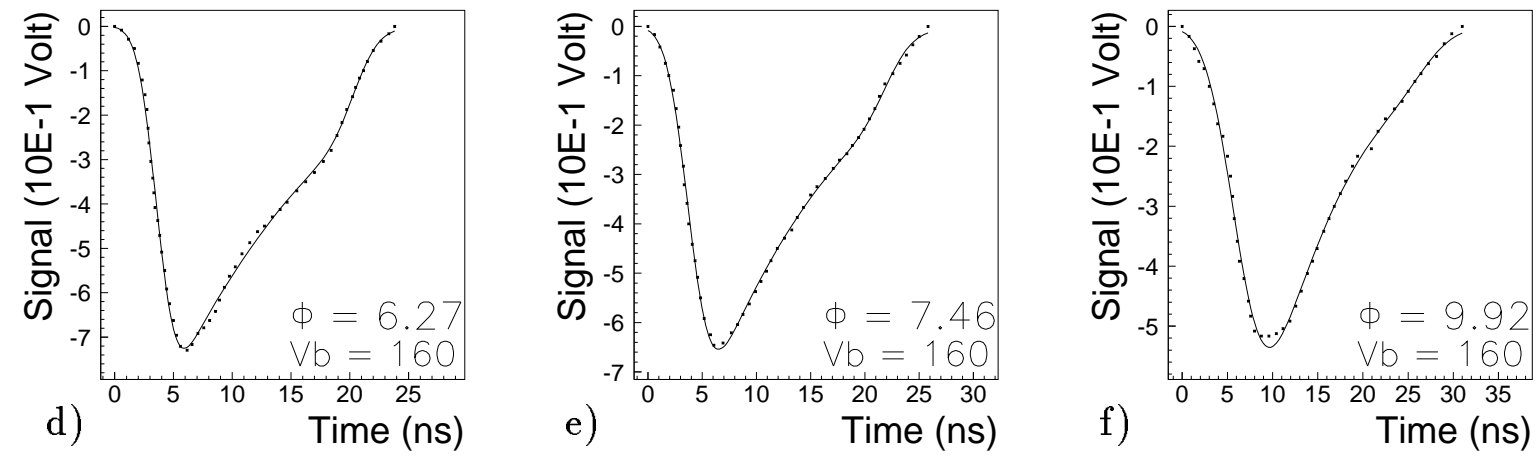

Fig. 4. 

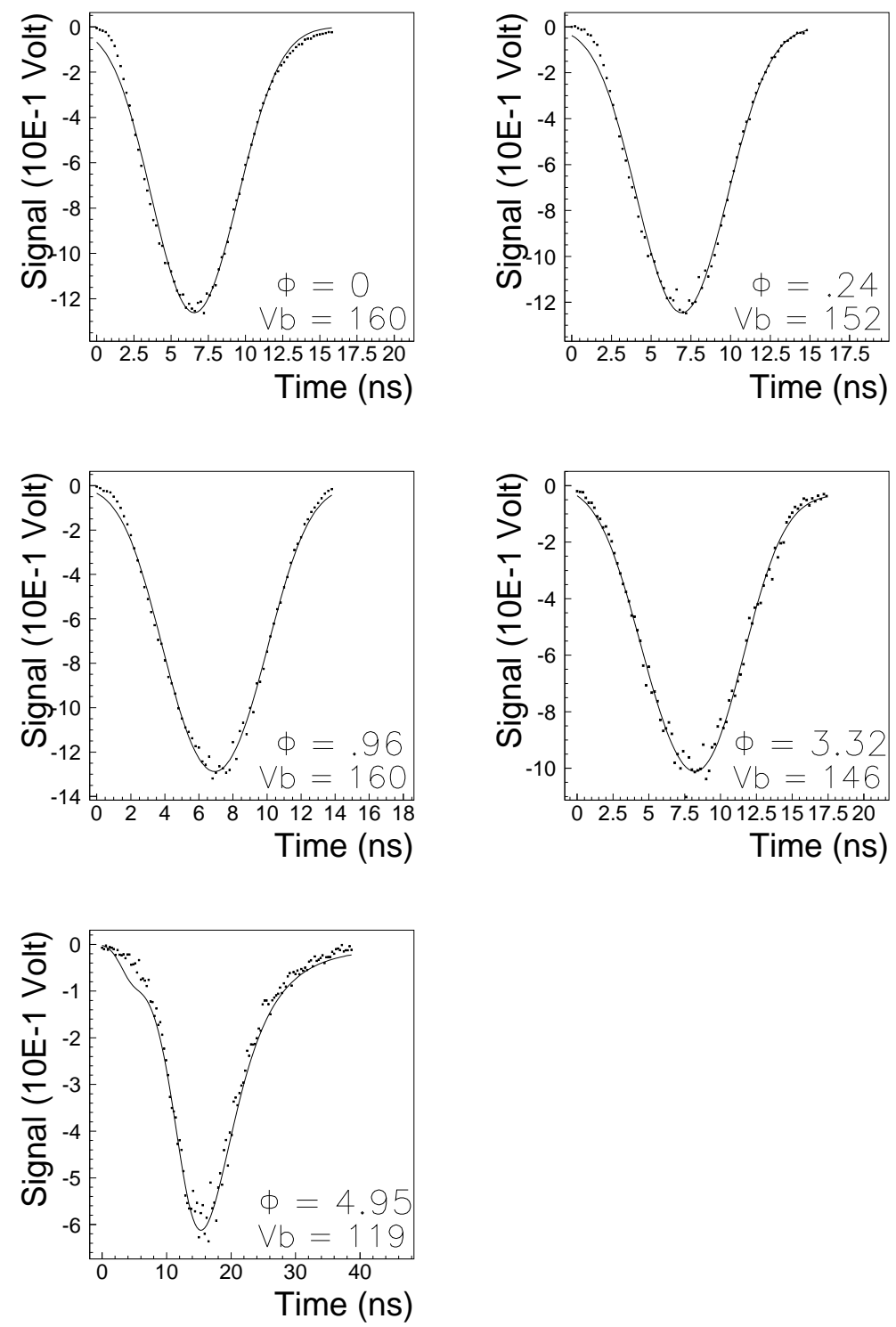

Fig. 5. 

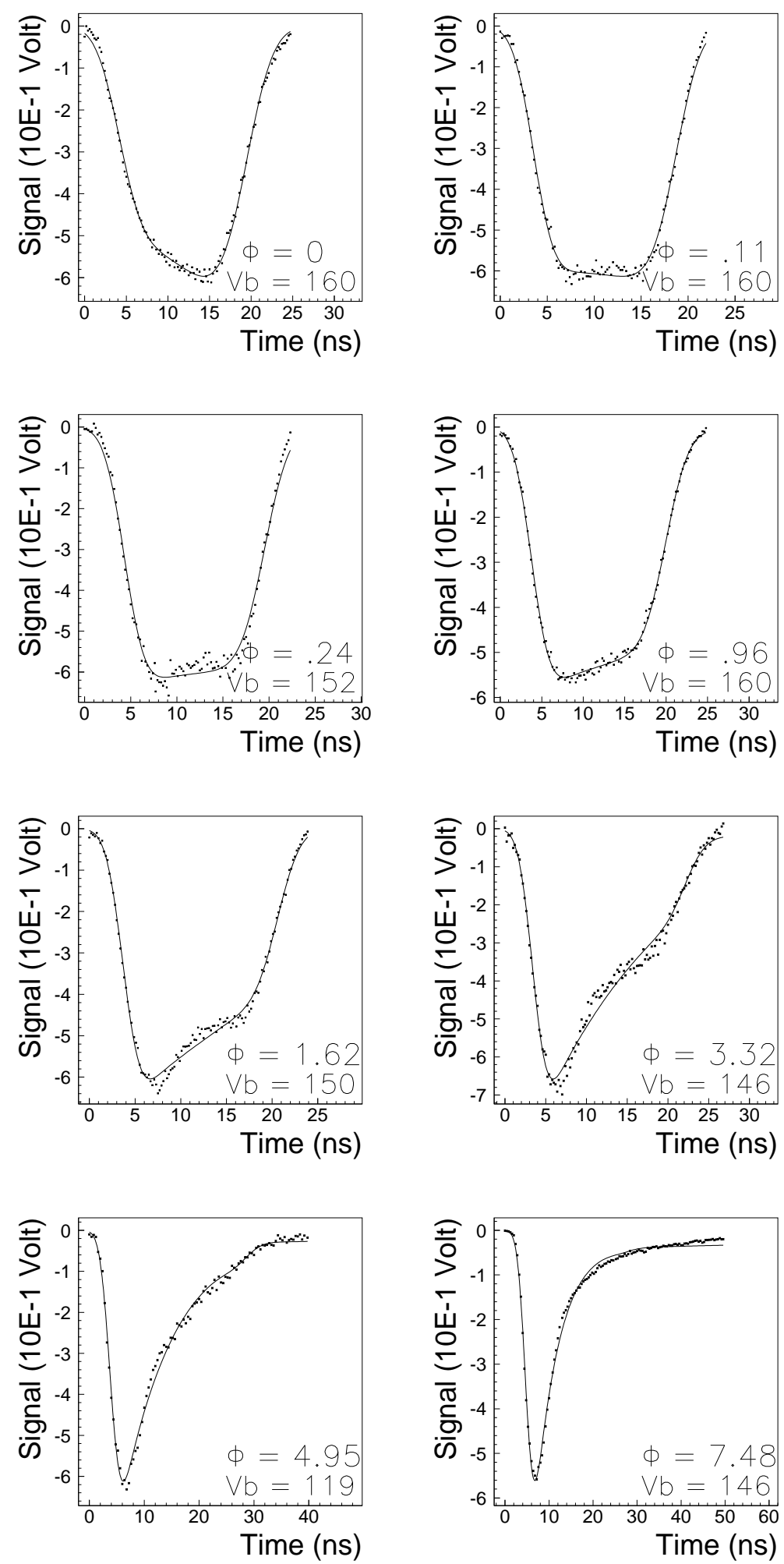

Fig. 6 . 

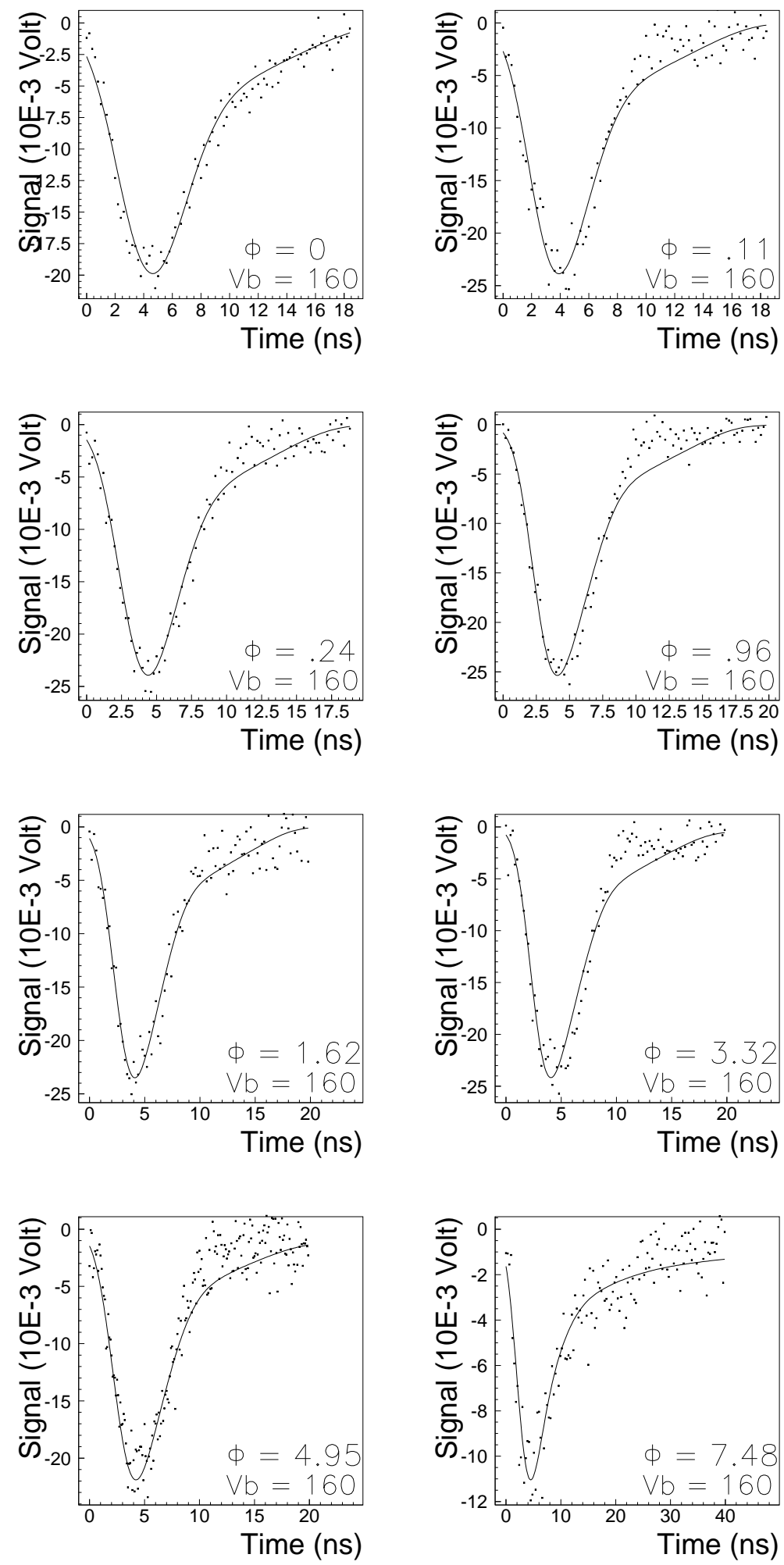

Fig. 7. 

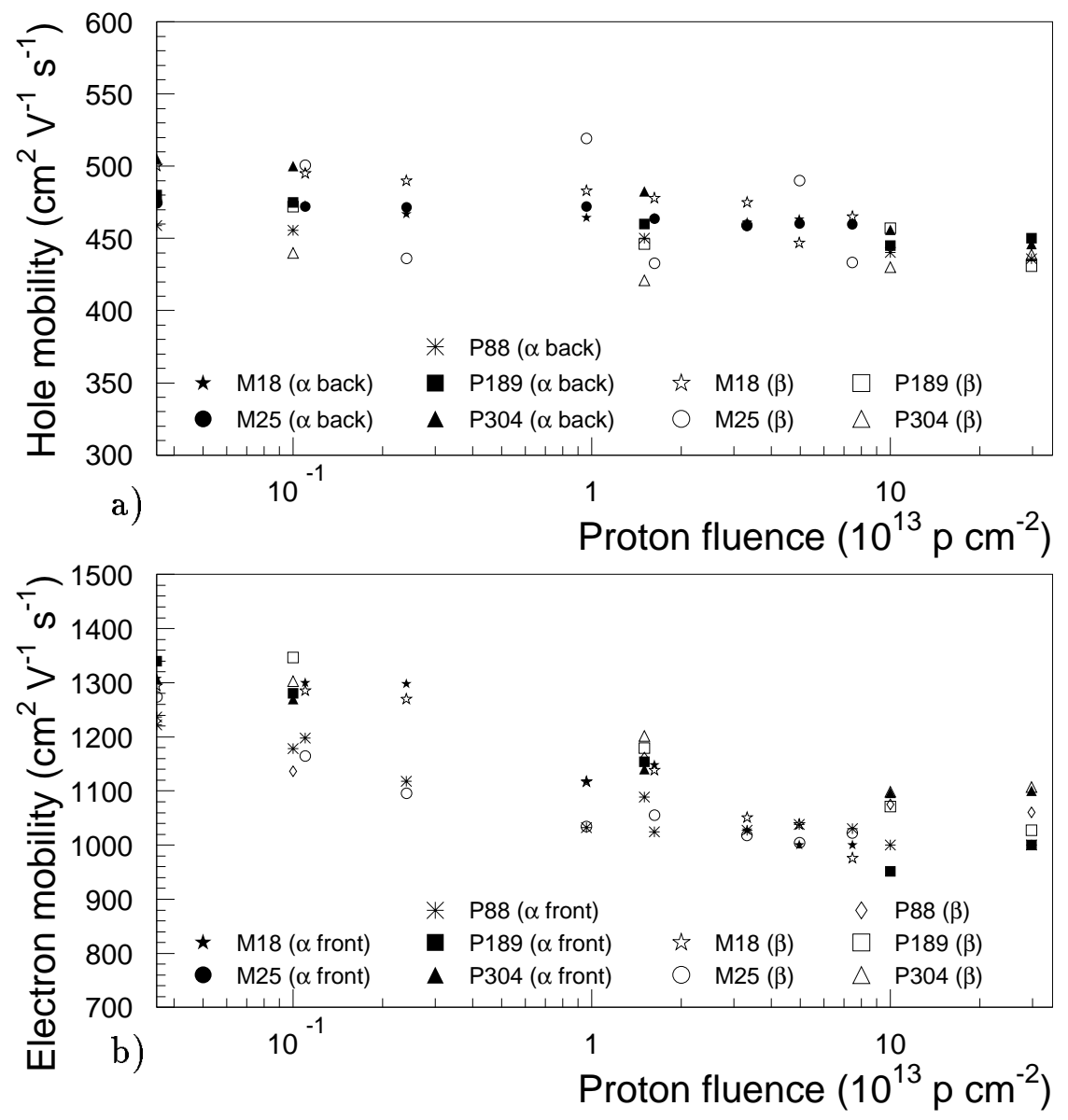

Fig. 8 . 